\title{
Resistência à penetração de um Latossolo Vermelho Amarelo do Cerrado sob diferentes sistemas de manejo
}

\author{
Ricardo Ralisch', Tiago M. Miranda ${ }^{2}$, Ricardo S. Okumura ${ }^{2}$, Graziela M. de C. Barbosa ${ }^{3}$, \\ Maria de F. Guimarães ${ }^{1}$, Eric Scopel ${ }^{4}$ \& Luiz C. Balbino ${ }^{5}$
}

\begin{abstract}
RESUMO
Embora associada à adoção de tecnologias de conservação do solo, a intensificação dos sistemas de produção agropecuária tem induzido a um dos principais problemas enfrentados pelos agricultores, a compactação do solo, seja em sistemas de plantio direto ou em sistemas de plantio convencional e pastagem. Este estudo foi realizado em Rio Verde, GO, em Latossolo Vermelho Amarelo, nos seguintes sistemas: plantio direto há 14 anos (SD14); plantio direto há oito anos (SD8); plantio direto há dois anos (SD2); preparo convencional (PC); pastagem (P) e floresta (F). A resistência à penetração do solo foi determinada utilizando-se o penetrômetro de Impacto Modelo IAA/Planalsucar - Stolf, a partir da superfície do solo até a profundidade de 0,40 m; determinaram-se, também, a densidade do solo e a granulometria. O plantio convencional apresentou menor resistência à penetração, na profundidade $0-0,10 \mathrm{~m}$, enquanto o SD2 indicou maior resistência à penetração até $0,40 \mathrm{~m}$ de profundidade. Abaixo da profundidade de $0,15 \mathrm{~m}$ não houve diferença significativa entre os tratamentos PC, SD8, SD14. Os dois primeiros anos de adoção do sistema de plantio direto são críticos quanto à resistência a penetração nas camadas superficiais do solo.
\end{abstract}

Palavras-chave: compactação do solo, plantio direto, plantio convencional

\section{Penetration resistance of a Red-Yellow Latosol of Cerrado under different management systems}

\begin{abstract}
The intensification of agricultural production systems has caused soil compaction even when soil conservation technologies are adopted. Soil compaction is one of the main problems for farmers, both in no-tillage and in conventional-tillage systems, as well as in pasture. This study was conducted in Rio Verde, (State of Goias), on Red-Yellow Latosol under the following systems: no-tillage for 14 years (SD14); no-tillage for 8 years (SD8); no-tillage for 2 years (SD2); conventional tillage (CT); pasture (P) and forest $(\mathrm{F})$. The soil resistance to penetration was measured by the impact penetrometer Model IAA/Planalsucar - Stolf, from soil surface to the soil depth of $0.40 \mathrm{~m}$. Soil bulk density and granulometry were also determined. Conventional tillage had lower resistance to penetration at the $0-0.10 \mathrm{~m}$ depth, whereas SD2 had more resistance up to $0.40 \mathrm{~m}$ in depth. There were no significant differences below $0.15 \mathrm{~m}$ in depth among treatments $\mathrm{CT}$, SD8, and SD14. The first two years under the no-tillage system are critical, with regard to the resistance to penetration in to the soil surface layers.
\end{abstract}

Key words: soil compaction, no-tillage, conventional tillage

\footnotetext{
1 Departamento de Agronomia, UEL, CP 6001, CEP 86051-990 Londrina, PR. Fone: (43) 3371-4555. E-mail: ralisch@uel.br; mfatima@uel.br

2 Graduando do Curso de Agronomia da Universidade Estadual de Londrina

3 IAPAR - Instituto Agronômico do Paraná. Fone: (43) 3376-2000. E-mail: graziela barbosa@iapar.br

${ }^{4}$ CIRAD PERSYST/Embrapa Cerrados. Fone: (61) 3388-9849. E-mail: eric.scopel@cirad.fr

${ }^{5}$ Embrapa Transferência de Tecnologia. Fone: (61) 3448-4570. E-mail: luizcarlos.balbino@embrapa.br
} 


\section{INTRODUÇÃO}

A adoção de novas tecnologias implantadas visando ao aumento da produtividade, normalmente induz a uma intensificação dos sistemas de produção agropecuária, que podem levar a diferentes formas de degradação do solo em médio e longo prazos, mesmo associadas às técnicas de conservação do solo. Depara-se, hoje, com um dos principais problemas enfrentados pelos agricultores em diversas regiões, que são os níveis de compactação do solo, tanto em sistemas de plantio direto como nos de plantio convencional e pastagem.

Esta compactação do solo ocorre quando este é submetido a determinada pressão, ocasionando redução de volume com conseqüente aumento da resistência à penetração e da densidade (Stone et al., 2002). Segundo Figueiredo et al. (2000), a compactação é uma conseqüência direta do manejo inadequado e a umidade é o fator que controla a quantidade de deformação que poderá ocorrer no solo. De maneira geral, a compactação altera as propriedades físicas do solo, comumente com o aumento da resistência mecânica à penetração e densidade do solo (Bertol et al., 2000), limita o crescimento vegetativo e compromete o rendimento das culturas (Dias Junior, 2000).

A penetrometria ou avaliação da resistência do solo à penetração é uma metodologia interessante para se avaliar, comparativamente, a variabilidade estrutural do perfil do solo (Ralisch \& Tavares Filho, 2002); apesar de não ser conclusivo quanto à intensidade e ocorrência de compactação do solo, permite avaliar diferentes sistemas empregados em mesmo tipo de solo, como no caso em questão.

O sistema de plantio direto demanda menor força de trabalho e de energia, estimula os processos de floculação e de agregação, reduz a velocidade de mineralização da matéria orgânica (Castro Filho et al., 1998), minimiza a erosão (Seganfredo et al., 1997) mas, em contrapartida, favorece o aparecimento da compactação decorrente do não revolvimento do solo e do excessivo tráfego de máquinas e implementos agrícolas (De Maria et al., 1999; Tormena et al., 1998).

No preparo convencional do solo com implementos agrícolas ocorrem alterações nas propriedades físicas, dependendo da intensidade de preparo do solo, em que as principais alterações são evidenciadas pelo aumento da resistência a penetração e, conseqüentemente na densidade do solo, taxa de infiltração de água, tamanho de agregados e volume de macroporos (Cavenage et al., 1999; Beutler et al., 2001). Considera-se, por outro lado, que a consolidação do sistema de plantio direto minimiza este efeito de compactação em função da proteção física da cobertura permanente, do aumento dos teores de matéria orgânica do solo e do efeito dos sistemas radiculares das plantas. Este estudo visa comparar diferentes períodos de adoção do sistema de plantio direto com sistema convencional, pastagem e floresta, tendo como referência este último, para analisar o comportamento da resistência do solo à penetração nesses sistemas, na região do Cerrado de Goiás.

\section{MATERIAL E MÉTODOS}

O estudo foi realizado em um Latossolo Vermelho Ama- relo, no sudoeste de Goiás, nas proximidades da cidade de Rio Verde, GO, coordenadas geográficas $17^{\circ} 48^{\prime}$ de latitude $\mathrm{S}$ e $50^{\circ} 55^{\prime}$ de longitude $\mathrm{W}$, altitude média de $715 \mathrm{~m}$ e clima tropical de Savana, segundo classificação de Köppen, do tipo AW. A topografia da região é plana a suavemente ondulada, com pendentes longas e declividades próximo de 3\%.

As áreas estudadas se encontravam com os seguintes tipos de manejo de solo: plantio direto há 14 anos (SD14); plantio direto há 8 anos (SD8); plantio direto há 2 anos (SD2); preparo convencional (PC), pastagem (P) e floresta (F).

Na área de plantio direto foram coletados dados de penetrometria durante o desenvolvimento da cultura do milho enquanto na área de plantio convencional se efetuou a coleta logo após a aração e gradagem, ambas na segunda quinzena de abril/04.

A resistência à penetração do solo foi determinada utilizando-se o penetrômetro de Impacto Modelo IAA/Planalsucar - Stolf, a partir da superfície do solo até a profundidade de $0,40 \mathrm{~m}$, em 15 pontos por parcela, segundo a metodologia descrita em Stolf (1991). A densidade do solo média nos perfis, avaliada pelo método de torrões e a granulometria, foram determinadas conforme EMBRAPA (1997).

Na Tabela 1 encontra-se a caracterização física das áreas estudadas.

Tabela 1. Granulometria e densidade do solo em diferentes sistemas de manejo em Latossolo Vermelho Amarelo

\begin{tabular}{|c|c|c|c|c|c|}
\hline \multirow{2}{*}{$\mathrm{SM}^{*}$} & \multicolumn{3}{|c|}{ Granulometria $\left(\mathrm{g} \mathrm{kg}^{-1}\right)$} & \multirow{2}{*}{ Classificação } & \multirow{2}{*}{$\begin{array}{l}\text { Densidade do } \\
\text { solo }\left(\mathrm{kg} \mathrm{dm}^{-3}\right)\end{array}$} \\
\hline & Argila & Silte & Areia & & \\
\hline SD14 & 439,40 & 142,50 & 418,10 & Argila & 1,01 \\
\hline SD8 & 601,60 & 102,80 & 295,60 & Argila & 1,13 \\
\hline SD2 & 576,60 & 127,80 & 295,60 & Argila arenosa & 1,15 \\
\hline PC & 561,06 & 126,66 & 312,26 & Argila & 1,16 \\
\hline$P$ & 611,60 & 102,80 & 285,60 & Muito argiloso & 1,07 \\
\hline $\mathrm{F}$ & 496,60 & 111,13 & 392,26 & Argila & 1,04 \\
\hline
\end{tabular}

$\mathrm{P}$ - Pastagem e F - Floresta

A umidade do solo tomada como média nos perfis dos tratamentos PC, SD8, SD14, P e F, variou entre 25 e $26 \%$, enquanto no SD2 a umidade média no perfil foi de $18 \%$.

Os resultados de resistência à penetração foram submetidos a análise de variância e as médias comparadas pelo teste de Tukey a $5 \%$ de probabilidade. Os dados foram transformados, quando necessário, segundo a metodologia de Box e Cox, descrita por Barbin (2003).

\section{RESULTADOS E DISCUSSÃO}

A Tabela 2 apresenta os dados de resistência do solo à penetração nos diferentes sistemas de manejo estudados; esses dados mostram que na profundidade de $0,05 \mathrm{~m}$ os sistemas de manejo SD2, SD8 e pastagem apresentaram os maiores valores e a menor resistência foi observada no PC. Comparando-se os sistemas de plantio direto, o SD14 indicou o menor valor de resistência à penetração; já na profundidade de 0,10 até 0,35 m, o SD2 apresentou o maior 
valor de resistência à penetração, estatisticamente diferente dos demais porém, comparando-se os sistemas SD8 e SD14, notou-se não serem diferentes significativamente nas profundidades de 0,10 até $0,40 \mathrm{~m}$; na profundidade $0,10 \mathrm{~m}$, a menor resistência foi observada no PC e floresta, enquanto para a profundidade 0,15 a $0,20 \mathrm{~m}$ os sistemas SD14, PC, pastagem e floresta, não apresentaram diferença significativa, obtendo-se os menores valores de resistência à penetração; por sua vez, os sistemas SD14, SD8, PC e pastagem, com valores em torno de 3,5 MPa, não apresentaram diferença significativa de resistência à penetração na profundidade de $0,25 \mathrm{~m}$; abaixo de $0,30 \mathrm{~m}$ somente a pastagem e a floresta possuem os menores valores de resistência à penetração.

Tabela 2. Resistência à penetração em diferentes sistemas de manejo (S.M.) nos Latossolo Vermelho Amarelo

\begin{tabular}{lllllllll}
\hline & \multicolumn{7}{c}{ Resistência a penetração } & $\mathbf{( M P a})^{* *}$ \\
\cline { 2 - 9 } & $\mathbf{0 , 0 5}$ & $\mathbf{0 , 1 0}$ & $\mathbf{0 , 1 5}$ & $\mathbf{0 , 2 0}$ & $\mathbf{0 , 2 5}$ & $\mathbf{0 , 3 0}$ & $\mathbf{0 , 3 5}$ & $\mathbf{0 , 4 0}$ \\
SD14 & $1,53 \mathrm{~b}$ & $2,24 \mathrm{bc}$ & $2,69 \mathrm{bc}$ & $3,25 \mathrm{bc}$ & $3,48 \mathrm{~b}$ & $3,59 \mathrm{bc}$ & $3,18 \mathrm{~b}$ & $3,10 \mathrm{bc}$ \\
SD8 & $2,80 \mathrm{a}$ & $2,73 \mathrm{~b}$ & $2,88 \mathrm{~b}$ & $3,40 \mathrm{~b}$ & $3,63 \mathrm{~b}$ & $3,33 \mathrm{bc}$ & $3,40 \mathrm{~b}$ & $2,80 \mathrm{c}$ \\
SD2 & $3,21 \mathrm{a}$ & $4,75 \mathrm{a}$ & $5,69 \mathrm{a}$ & $6,36 \mathrm{a}$ & $6,06 \mathrm{a}$ & $5,50 \mathrm{a}$ & $4,64 \mathrm{a}$ & $4,08 \mathrm{a}$ \\
PC & $0,70 \mathrm{c}$ & $1,41 \mathrm{~d}$ & $2,58 \mathrm{bc}$ & $3,03 \mathrm{bc}$ & $3,93 \mathrm{~b}$ & $4,00 \mathrm{~b}$ & $3,40 \mathrm{~b}$ & $3,48 \mathrm{ab}$ \\
P & $3,98 \mathrm{a}$ & $2,73 \mathrm{~b}$ & $2,73 \mathrm{bc}$ & $3,03 \mathrm{bc}$ & $3,10 \mathrm{~b}$ & $3,03 \mathrm{~cd}$ & $2,73 \mathrm{bc}$ & $2,73 \mathrm{~cd}$ \\
F & $1,38 \mathrm{~b}$ & $1,60 \mathrm{~cd}$ & $2,05 \mathrm{c}$ & $2,50 \mathrm{c}$ & $2,13 \mathrm{c}$ & $2,43 \mathrm{~d}$ & $2,05 \mathrm{c}$ & $2,13 \mathrm{~d}$ \\
C.V. & 15,39 & 8,77 & 7,04 & 1,93 & 7,58 & 13,68 & 7,24 & 25,18 \\
\hline
\end{tabular}

* Plantio direto há 14 anos (SD 14), 8 anos (SD 8), 2 anos (SD2), PC - Preparo Convencional, $\mathrm{P}$ - Pastagem e F - Floresta; ${ }^{* *}$ Médias seguidas das mesmas letras nas colunas não diferem entre si pelo teste de Tukey $(P<0,05)$

Em sistemas de plantio convencional, em que a camada superficial do solo é constantemente revolvida, é comum observar aumento de sua macroporosidade, motivo pelo qual a área submetida ao preparo convencional apresentou menor resistência à penetração $(0,70 \mathrm{MPa})$ na superfície do solo $(0,05 \mathrm{~m})$, haja vista que a coleta dos dados foi realizada pouco tempo após a aração e gradagem da área. Resultados semelhantes foram obtidos por Prado et al. (2002), Tormena et al. (2002) e De Maria et al. (1999). A pastagem apresentou maior valor de resistência à penetração na superfície devido, provavelmente, ao pisoteio dos animais; enfim, o sistema SD14 mostrou valores semelhantes aos do solo da floresta até a profundidade de 0,20 m, em função da melhoria da estrutura do solo em razão do longo período de implantação do sistema de plantio direto, sem qualquer revolvimento do solo.

Na profundidade de 0,10 e 0,15 m, os valores relativos ao plantio convencional (PC) são estatisticamente comparáveis aos da floresta (F), não justificando a realização da aração visto que o objetivo desta operação é aumentar a porosidade, reduzir a densidade e a resistência a penetração do solo.

Após esta camada se percebe o efeito do acúmulo das cargas dos implementos de preparo de solo utilizados, formando o chamado "pé de grade” (Tormena et al., 1998; Derpsch et al., 1991) que resulta no aumento da resistência a penetração; este efeito ficou evidente pois a resistência a penetração se manteve superior a $3 \mathrm{MPa}$.

Os sistemas PC, SD8, SD14 e a pastagem, na profundi- dade de 0,15 m e 0,20 m, apresentaram valores de resistência à penetração estatisticamente iguais, contradizendo os resultados de Moraes \& Benez (1996), que obtiveram maiores valores de resistência à penetração nesta mesma profundidade nas áreas submetidas a aração e gradagem, em relação ao plantio direto, denotando que a variação das condições do solo no momento das operações de preparo altera os resultados de resistência do solo a penetração.

A área em que se praticou, durante dois anos, o plantio direto (SD2), foi a que apresentou maior valor de resistência à penetração (acima de $4 \mathrm{Mpa}$ ) a partir de 0,10 m, devido, provavelmente, a estruturas mais densas do solo oriundas do sistema convencional anterior e inadequadamente corrigidas para implantação do sistema de plantio direto (Ralisch \& Tavares Filho, 2002) e ao menor teor de umidade deste solo (18\%). Figueiredo et al. (2000), concluíram ser a compactação conseqüência de manejos inadequados, do resultado do não revolvimento do solo na camada subsuperficial e do tráfego de máquinas (Beutler et al., 2001).

Valores elevados de resistência à penetração na camada superficial do solo, em plantio direto, também foram observados por Tavares Filho et al. (2001) e Stone \& Silveira (2001); contudo, esses valores não chegaram a comprometer o sistema, em razão da maior continuidade dos poros, homogeneidade do solo e maior atividade microbiana (Silva et al., 2000).

Avaliando-se os valores de resistência à penetração nos três sistemas de plantio direto, observa-se redução desses valores, praticamente em todas as profundidades, conforme os anos de implantação do sistema de plantio direto, fato este dependente dos benefícios que tal sistema proporciona ao solo (Pedrotti et al., 2001), como o acúmulo de matéria orgânica em superfície (lenta mineralização), associado à macro e microfaunas, que desempenham papel significativo nas propriedades físicas do solo (Castro Filho et al., 1998).

A densidade do solo exerce papel fundamental nesta relação, uma vez que os sistemas de preparo alteram a densidade do solo, constatando-se seu aumento em sistemas de plantio direto, além da sua redução em preparos convencionais (Secco et al., 2005; Watanabe et al., 2002). Corsini \& Ferraudo (1999) observaram aumento nos valores da resistência do solo à penetração e alterações na distribuição de poros em sistema de plantio direto. Os efeitos do tráfego de máquinas e implementos agrícolas no aumento da densidade também têm sido demonstrados por vários autores, como De Maria et al. (1999), Tormena et al. (1998) e Beutler et al. (2001).

Observou-se, neste estudo, que os anos de implantação da plantio direto resultaram em redução da resistência do solo à penetração podendo ser este um dos fatores de aumento da produtividade com a consolidação do sistema.

\section{CONCLUSÕES}

1. O plantio convencional apresentou os menores valores de resistência à penetração, na profundidade 0-0,10 m. 
2. Os perfis de resistência do solo à penetração que mais se aproximaram do solo natural, foram os dos sistemas pastagem (P) e plantio direto com 14 anos (SD14).

\section{LITERATURA CITADA}

Barbin, D. Planejamento e análise estatística de experimentos agronômicos. 1.ed. Arapongas: Midas, 2003. p.194.

Bertol, I.; Schick, J.; Massariol, J. M.; Reis, E. F. dos; Dilly, L. Propriedades físicas de um Cambissolo Húmico álico afetadas pelo manejo do solo. Ciência Rural, v.30, n.1, p.91-95, 2000.

Beutler, A. N.; Silva, N. L. N.; Curi, N.; Ferreira, M. M.; Cruz, J. N.; Pereira Filho, I. A. Resistência a penetração e permeabilidade de Latossolo Vermelho Distrófico típico sob sistemas de manejo na região dos cerrados. Revista Brasileira de Ciência do Solo, v.25, n.1 p.167-177, 2001.

Castro Filho, C.; Muzilli, O.; Padanoschi, A. L. Estabilidade dos agregados e sua relação com o teor de carbono orgânico num Latossolo Roxo distrófico, em função de sistemas de plantio, rotações de culturas e métodos de preparo das amostras. Revista Brasileira de Ciência do Solo, v.22, n.3 p.527-538, 1998.

Cavenage, A.; Moraes, M. L. T.; Alves, M. C.; Carvalho, M. A. C.; Freitas, M. L. M.; Buzetti, S. Alterações nas propriedades físicas de um Latossolo Vermelho-Escuro sob diferentes culturas. Revista Brasileira de Ciência do Solo, v.23, n.4, p.9971003, 1999.

Corsini, P. C.; Ferraudo, A. S. Efeitos de sistemas de cultivo na densidade e macroporosidade do solo e no desenvolvimento radicular do milho em Latossolo Roxo. Pesquisa Agropecuária Brasileira, v.34, p.289-298, 1999.

De Maria, I. C.; Castro, O. M.; Dias, H. S. Atributos físicos do solo e crescimento radicular de soja em Latossolo Roxo sob diferentes métodos de preparo do solo. Revista Brasileira de Ciência do Solo, v.23, n.3, p.703-709, 1999.

Derpsch, R.; Roth, C.; Sidiras, N.; Köpke, U. Controle de erosão no Paraná, Brasil: Sistemas de cobertura do solo, plantio direto e preparo conservacionista do solo. Eschborn: Deutsche Gesellschaff für Technische Zusammenarbeit (GTZ), 1991. 272p.

Dias Junior, M. S. Compactação do solo. In: Tópicos em ciência do solo. Sociedade Brasileira de Ciência do Solo, v.1, p.55-94, 2000.

EMBRAPA - Empresa Brasileira de Pesquisa Agropecuária. Centro Nacional de Pesquisa de Solos. Sistema brasileiro de classificação de solos. Brasília: Embrapa Produção de Informação; Rio de Janeiro: Embrapa Solos, 1997. 412p.

Figueiredo, L. H. A.; Dias Junior, M. S.; Ferreira, M. M. Umidade crítica de compactação e densidade do solo máxima em resposta a sistemas de manejo num Latossolo Roxo. Revista Brasileira de Ciência do Solo, v.24, n.3, p.487-493, 2000.

Moraes, M. H.; Benez, S. H. Efeitos de diferentes sistemas de preparo do solo em algumas propriedades físicas de uma terra roxa estruturada e na produção de milho para um ano de cultivo. Engenharia Agrícola, v.16, n.2, p.31-41, 1996.
Pedrotti, A.; Pauletto, E. A; Crestana, S.; Ferreira, M. M.; Dias Junior, M. S.; Gomes, A. S.; Turatti, A. L. Resistência mecânica à penetração de um Planossolo submetido a diferentes sistemas de cultivo. Revista Brasileira de Ciência do Solo, v.25, n.3, p.521-529, 2001.

Prado, R. M.; Roque, C. M.; Souza, Z. M. Sistemas de preparo e resistência a penetração e densidade de um Latossolo Vermelho eutrófico em cultivo intensivo e pousio. Pesquisa Agropecuária Brasileira, v.37, p.1795-1801, 2002.

Ralisch, R; Tavares Filho, J. Compactação do solo em sistema de plantio direto - causas, efeitos e prevenção. In: Encontro Nacional de Plantio Direto na Palha, 8, 2002, Águas de Lindóia. Resumos... Ponta Grossa: Federação Brasileira de Plantio Direto na Palha, 2002. p.125-127.

Secco, D.; Ros, C. O.; Secco, J. K.; Fiorin, J. E. Atributos físicos e produtividade de culturas em um Latossolo vermelho argiloso sob diferentes sistemas de manejo. Revista Brasileira de Ciência do Solo, v.29, n.3, p.407-414, 2005.

Seganfredo, M. L.; Eltz, F. L. F.; Brum, A. C. R. Perdas de solo, água e nutrientes por erosão em sistemas de culturas em plantio direto. Revista Brasileira de Ciência do Solo, v.21, n.2, p.287-291, 1997.

Silva, V. R.; Reinert, D. J.; Reichert, J. M. Suscetibilidade à compactação de um Latossolo Vermelho-Escuro e de um Podzólico Vermelho-Amarelo. Revista Brasileira de Ciência do Solo, v.24, n.2, p.239-249, 2000.

Stolf, R. Teoria e teste experimental de fórmulas de transformação dos dados de penetrômetro de impacto em resistência do solo. Revista Brasileira de Ciência do Solo, v.15, n.2, p.229-235, 1991.

Stone, L. F.; Guimarães, C. M.; Moreira, J. A. A. Compactação do solo na cultura do feijoeiro - I Efeitos nas propriedades físico-hídricas do solo. Revista Brasileira de Engenharia Agrícola e Ambiental, v.6, n.2, p.207-212, 2002.

Stone, L. F.; Silveira, P. M. Efeitos do sistema de preparo e da rotação de culturas na porosidade e densidade do solo. Revista Brasileira de Ciência do Solo, v.25, n.2, p.395-401, 2001.

Tavares Filho, J.; Barbosa, G. M. C.; Guimarães, M. F.; Fonseca, I. C. B. Resistência do solo à penetração e desenvolvimento do sistema radicular do milho (Zea mays) sob diferentes sistemas de manejo em um Latossolo Roxo. Revista Brasileira de Ciência do Solo, v.25, n.3, p.725-730, 2001.

Tormena, C. A.; Barbosa, M. C.; Costa, A. C. S. Densidade, porosidade e resistência a penetração em latossolo cultivado sob diferentes sistemas de preparo do solo. Sciencia Agrícola, v.59, n.4, p.795-801, 2002.

Tormena, C. A.; Roloff, G.; Sá, J. C. M. Propriedades físicas do solo sob plantio direto influenciadas por calagem, preparo inicial e tráfego. Revista Brasileira de Ciência do Solo, v.22, n.2, p.301-309, 1998.

Watanabe, S. H.; Tormena, S. A.; Araujo, M. A.; Vidigal Filho, P. S.; Pintro, J. C.; Costa, A. C. S.; Muniz, A. S. Propriedades físicas de um Latossolo Vermelho distrófico influenciadas por sistemas de preparo do solo utilizados para implantação da cultura de mandioca. Acta Scientiarun, v.24, n.5, p.1255-1264, 2002. 